PROCEEDINGS OF THE

AMERICAN MATHEMATICAL SOCIETY

Volume 131, Number 10, Pages 3113-3121

S 0002-9939(03)06898-9

Article electronically published on February 6, 2003

\title{
ILL-POSEDNESS FOR THE ZAKHAROV SYSTEM WITH GENERALIZED NONLINEARITY
}

\author{
H. A. BIAGIONI AND F. LINARES \\ (Communicated by David S. Tartakoff)
}

\begin{abstract}
We study the ill-posedness question for the one-dimensional Zakharov system and a generalization of it in one and higher dimensions. Our point of reference is the criticality criteria introduced by Ginibre, Tsutsumi and Velo (1997) to establish local well-posedness.
\end{abstract}

\section{INTRODUCTION}

This note is concerned with the initial value problem associated to the Zakharov system

$$
\left\{\begin{array}{l}
i \partial_{t} u+\Delta u=n u, \quad x \in \mathbb{R}^{d}, t \in \mathbb{R}, \\
\square n=\Delta\left(|u|^{2}\right), \\
\left(u, n, \partial_{t} n\right)(0)=\left(u_{0}, n_{0}, n_{1}\right)
\end{array}\right.
$$

and generalizations of it (see (3.6)). The system in (1.1) appears as a model for the Langmuir turbulence in plasma (see [16]). Local and global well-posedness theory for the Zakharov system have been objects of extensive study by several authors in the last few years ([1], 2], [7], [9], 10, [12, [14]). Recently, Ginibre, Tsutsumi and Velo ([9]) have obtained local well-posedness results for low regularity data. This is the starting point of our study. We will focus on the one-dimensional case. Our purpose is to investigate the ill-posedness question for the initial value problem.

We give an example showing in what Sobolev spaces the associated initial value problem to the one-dimensional system (1.1) is indeed ill posed (Proposition 2.2). We use as a reference the criticality criteria given in 9 . In particular, our example shows that for the critical case, the IVP (1.1) is ill posed. This criticality criteria is not a standard one. Usually the criteria employed is the one given by a scaling argument. In [9] they modified the scaling argument due to the lack of homogeneity presented in the equations involved in the system (1.1).

We also deal with a generalization of (1.1) studied by Colliander in 8] (see (3.6) below). We give an example showing where ill-posedness has to occur. This result makes more precise the conditions guaranteeing the local well-posedness of this system (see Proposition 3.3).

Received by the editors June 15, 2001 and, in revised form, April 28, 2002.

2000 Mathematics Subject Classification. Primary 35Q55, 35Q51.

Key words and phrases. Ill-posedness, Zakharov system.

(C)2003 American Mathematical Society 
The notion of well-posedness we will use along this work is the following: existence, uniqueness, persistence property and instead of continuous dependence of the solution upon the data we will require the mapping data, $u_{0} \rightarrow u(t)$, being uniformly continuous, where $u(t)$ is the solution associated to the initial value problem. In case this last requirement is not satisfied we will say that the problem is ill posed.

Our approach is based on the ideas used by Kenig, Ponce and Vega [11 to show ill-posedness for the nonlinear Schrödinger, Korteweg-de Vries and modified Korteweg-de Vries equation. The main ingredient in their proof is the use of the invariance of the equations under some transformations and special solutions. Here we have to point out a previous work related to the ill-posedness for nonlinear dispersive equations. In [5] Birnir, Kenig, Ponce, Svanstedt and Vega established ill-posedness for the IVP associated to the generalized KdV. This result in particular shows the sharpness of the local well-posedness for that problem. In our case the latter method seems not to be applicable. The approach in [5] uses properties of the solitary wave solutions of the equation, say $\phi(x-c t)$. The existence of such special functions for any speed of propagation $c>0$ and the exponential decay are strongly applied. In [15] were found solitary wave solutions for the one-dimensional Zakharov system (2.2); however, the speed of propagation is restricted to a bounded interval. This fact prevents us from applying the argument in [5] for those solitary waves. The techniques in [11] and [5] have been applied sucessfully in other situations; see for instance [4].

In the next sections we will give an account of the results concerning the onedimensional Zakharov system and the Zakharov system with generalized nonlinearity. In each case we compare the existing results with ours. The last section will be devoted to show Propositions 2.2 and 3.3 .

\section{Results Regarding the 1D Zakharov system}

We consider the initial value problem associated to the one-dimensional Zakharov system

$$
\left\{\begin{array}{l}
i \partial_{t} u+\partial_{x}^{2} u=n u, \\
\square n=\partial_{x}^{2}\left(|u|^{2}\right), \\
\left(u, n, \partial_{t} n\right)(0)=\left(u_{0}, n_{0}, n_{1}\right)
\end{array}\right.
$$

where $u$ is a complex-valued function and $n$ a real-valued function.

Notion of criticality for the Zakharov system. For initial data $\left(u_{0}, n_{0}, n_{1}\right) \in$ $H^{k}\left(R^{d}\right) \oplus H^{\ell}\left(\mathbb{R}^{d}\right) \oplus H^{\ell-1}\left(\mathbb{R}^{d}\right)$, the critical values are

$$
k=\frac{d}{2}-\frac{3}{2} \quad \text { and } \quad \ell=\frac{d}{2}-2
$$

where $d$ denotes the dimension. Thus, in the one-dimensional case,

$$
k=-1 \quad \text { and } \quad \ell=-\frac{3}{2}
$$

see [9], p. 387. In particular the optimal relation between $k$ and $\ell$ is $k-\ell=\frac{1}{2}$.

In $[9]$ the following result in the one-dimensional case was proved. 
Proposition 2.1. The Zakharov system (2.2) is locally well posed for initial data $\left(u_{0}, n_{0}, n_{1}\right) \in H^{k}(\mathbb{R}) \oplus H^{\ell}(\mathbb{R}) \oplus H^{\ell-1}(\mathbb{R})$ provided

$$
-\frac{1}{2}<k-\ell \leq 1, \quad 2 k \geq \ell+\frac{1}{2} \geq 0 .
$$

The solutions satisfy

$$
\left(u, n, \partial_{t} n\right) \in C\left(H^{k} \oplus H^{\ell} \oplus H^{\ell-1}\right) .
$$

Note that the lowest allowed values of $(k, \ell)$ for fixed $k-\ell$ are obtained for $k-\ell=\frac{1}{2}$ and are given by $(k, \ell)=(0,-1 / 2)$.

In 9] a counterexample is provided showing that the second condition of (2.4) is necessary in the functional framework of [9. These results show a gap between the critical values in (2.3) and (2.4). We will prove that (2.2) is ill posed for some values in that gap.

In the one-dimensional case, we have the following result for ill-posedness:

Proposition 2.2. The Zakharov system (2.2) is ill posed in $H^{k} \oplus H^{\ell} \oplus H^{\ell-1}, k<0$ and $\ell \leq-3 / 2$.

Remark 2.3. The proposition above shows that for the critical values given in (2.3) the IVP (2.2) is ill posed, but there is still a gap between our result and the one given by $[9]$ as shown in Figure 1.

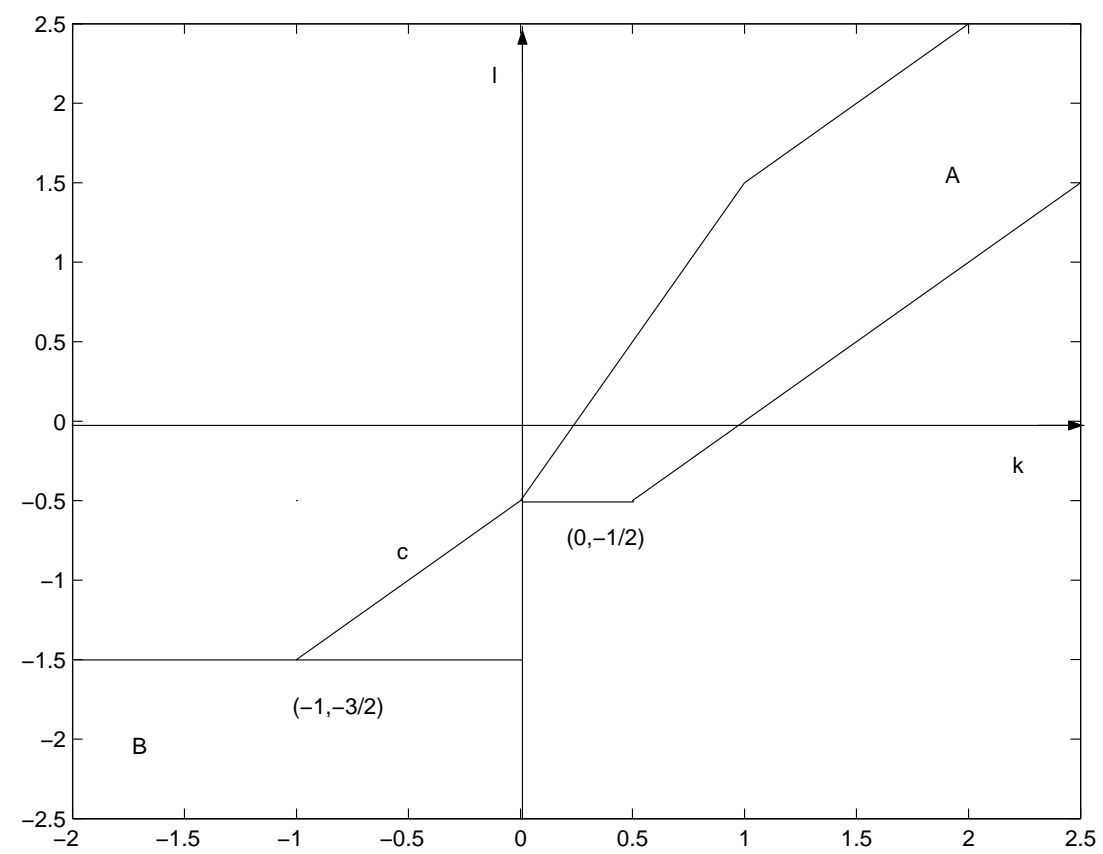

Figure 1. Local well-posedness was shown in [9] for indexes $(k, l)$ in region $\mathrm{A}$. Region $\mathrm{B}$ contains the indexes $(k, l)$ where ill-posedness is shown by our example. The line segment $c:=k-l=1 / 2$ contains the indexes where well-posedness is conjectured. 


\section{RESUlts REGARDing The ZAKHAROV SYSTEMS WITH GENERALIZED NONLINEARITY}

In [8] it was considered the IVP for the following generalization of the Zakharov system:

$$
\left\{\begin{array}{l}
i \partial_{t} u+\Delta u=m n|u|^{2(m-1)} u, \\
\square n=\Delta|u|^{2 m}, \\
\left(u, n, \partial_{t} n\right)(0)=\left(u_{0}, n_{0}, n_{1}\right) \in H^{s}\left(\mathbb{R}^{d}\right) \times H^{k}\left(\mathbb{R}^{d}\right) \times \hat{H}^{k-1}\left(\mathbb{R}^{d}\right)
\end{array}\right.
$$

denoted by $\left(Z S_{m}\right)$. Here $m \geq 1$ denotes an integer number.

The results in $[8$ are the following:

Theorem 3.1. $\left(Z S_{m}\right)$ on $\mathbb{R}^{d}, d=1,2$, is locally well posed in $H^{s_{d, m}} \times L^{2} \times \dot{H}^{-1}$ with $s_{d, m}<1$ for all $m \in \mathbb{N}$.

Theorem 3.2. Suppose for some $s \geq 1$, the initial data $\left(u_{0}, n_{0}, n_{1}\right)$ satisfies the regularity condition $\left(u_{0}, n_{0}, n_{1}\right) \in \bigcup_{\sigma<s} H_{\sigma}$. Then the solution $(u(t), n(t))$ to $\left(Z S_{m}\right)$, valid on the interval $[0, T)$ guaranteed by Theorem 3.1] also satisfies $(u(t), n(t)$, $\left.\partial_{t} n(t)\right) \in \bigcup_{\sigma<s} H_{\sigma}$ for $t \in[0, T)$.

Above $H_{\sigma}$ denotes the triple of function spaces $H^{\sigma} \times H^{\sigma-1} \times \hat{H}^{\sigma-2}$ and $\hat{H}^{s}$ denotes the space of distributions $\phi$ such that $\phi=\operatorname{div} V, V: \mathbb{R}^{d} \rightarrow \mathbb{R}^{d}$ and $\|\phi\|_{\hat{H}^{s}}=$ $\|V\|_{H^{s+1}}$.

Critical values for $\left(Z S_{m}\right)$. Following the criticality notion in 9], the (IVP) $\left(Z S_{m}\right)$ should be well posed in $H^{k}\left(\mathbb{R}^{d}\right) \times H^{\ell}\left(\mathbb{R}^{d}\right) \times H^{\ell-1}\left(\mathbb{R}^{d}\right)$ for $k>\$_{m, d}, \ell>$ $\$_{m, d}-\frac{1}{2}$ where

$$
\$_{m, d}=\frac{d}{2}-\frac{3}{4 m-2} .
$$

The result concerning ill-posedness for the system (3.6) is the following:

Proposition 3.3. The Zakharov system $\left(Z S_{m}\right)$ is ill posed in $H^{k}\left(\mathbb{R}^{d}\right) \times H^{\ell}\left(\mathbb{R}^{d}\right)$ $\times H^{\ell-1}\left(\mathbb{R}^{d}\right)$, if

$$
d>\frac{2}{2 m-1}, 0<k \leq \alpha_{m, d}:=\frac{d}{2}-\frac{1}{2 m-1}, \ell \leq \ell_{m, d}:=\frac{d}{2}-\frac{2 m}{2 m-1} .
$$

Remark 3.4. Notice that $\$_{m, d}<\alpha_{m, d}$ and $\ell_{m, d}<\$_{m, d}-\frac{1}{2}$. Then the region of ill-posedness is a rectangle $\left.\left.B:=] 0, \alpha_{m}\right] \times\right]-\infty, \ell_{m, d}[$ which is below the region of well-posedness suggested by Colliander in [8]. See Figure 2 .

Remark 3.5. If $d=2$ the range of $(k, \ell)$ is $0 \leq k \leq 1-\frac{1}{2 m-1}<1$ and $\ell \leq-\frac{1}{2 m-1}$, in agreement with the results in Theorem 3.1 although the number $s_{d, m}<1$ appearing there is not explicitely given.

\section{Proof of Propositions 2.2 and 3.3}

We begin this section by giving the proof of Proposition 3.3

Proof of Proposition 3.3. Let

$$
\left\{\begin{array}{l}
u_{\omega, N}(x, t)=e^{-i t\left(|N|^{2}-\omega^{2}\right)} e^{i N \cdot x} f_{\omega}(x-2 t N), \\
n_{\omega}(x, t)=-\left(f_{\omega}(x)\right)^{2 m}
\end{array}\right.
$$




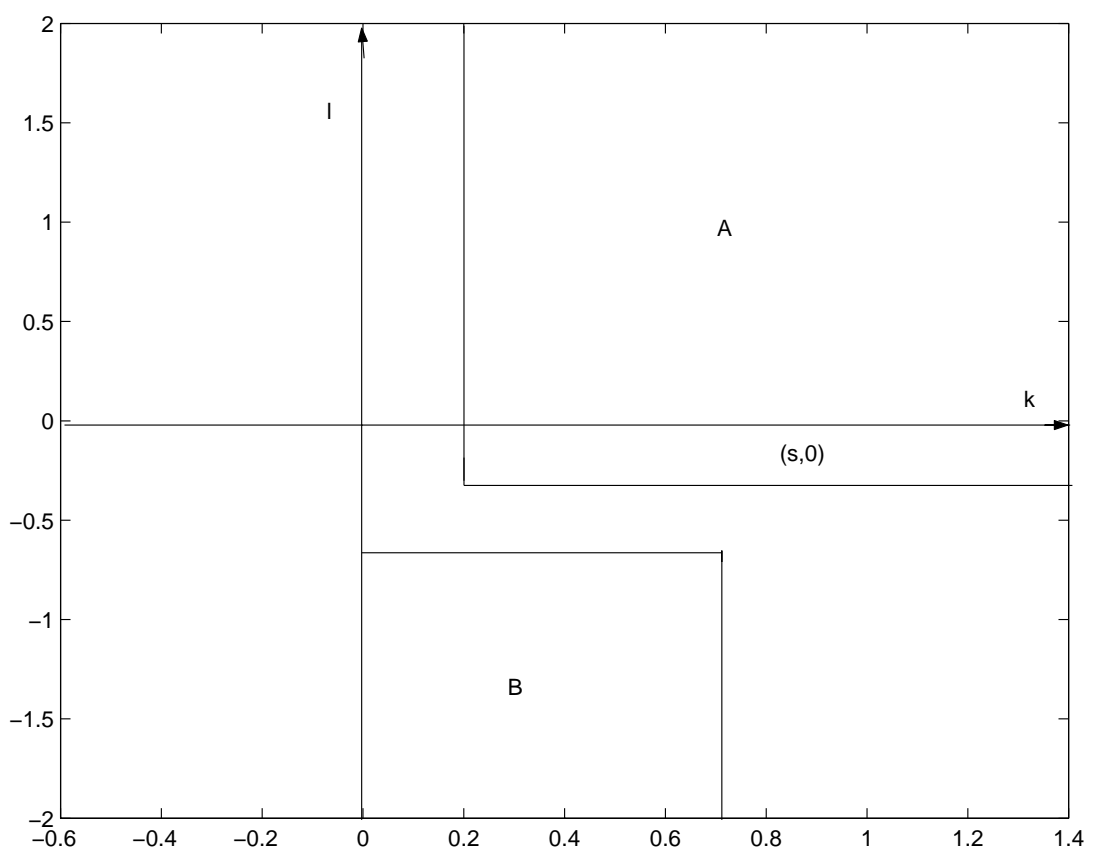

FIGURE 2.

The function $f$ above is the unique positive solution of $-f+\Delta f+|f|^{2(m-1)} f=0$. Solutions of this equation are radially symmetric and decay exponentially to zero as $|x| \rightarrow \infty$; see [3], [13]. In addition, the scaling

$$
f_{\omega}(x)=\omega^{\frac{2}{4 m-2}} f(\omega x)
$$

is a solution of

$$
-\omega^{2} f_{\omega}+\Delta f_{\omega}+\left(f_{\omega}\right)^{4 m-1}=0 .
$$

The pair (4.9) is a solution of $\left(Z S_{m}\right)$ with initial data $\left(e^{i N \cdot x} f_{\omega},-\left|f_{\omega}\right|^{2 m}, 0\right)$. In fact, by the Galilean invariance, $u_{\omega, N}$ is a solution of

$$
i \partial_{t} u+\Delta u+|u|^{4 m-2} u=0
$$

with initial data $u_{N, \omega}(x, 0)=e^{i N \cdot x} f_{\omega}$.

We have

$$
\begin{aligned}
\widehat{f_{\omega}}(\xi) & =\omega^{\frac{1}{2 m-1}-d} \hat{f}\left(\frac{\xi}{\omega}\right), \\
\mathcal{F}\left(f_{\omega}\right)^{2 m}(\xi) & =\omega^{\frac{2 m}{2 m-1}-d} \mathcal{F}\left(f^{2 m}\right)\left(\frac{\xi}{\omega}\right) .
\end{aligned}
$$


By the Taylor formula with integral remainder, we have, from (4.10),

$$
\begin{aligned}
& \left|\widehat{f_{\omega}}\left(\xi-N_{1}\right)-\widehat{f_{\omega}}\left(\xi-N_{2}\right)\right|^{2}=\left|\int_{0}^{1} \nabla \widehat{f_{\omega}}\left(\xi-N_{2}+t\left(N_{2}-N_{1}\right)\right) \cdot\left(N_{2}-N_{1}\right) d t\right|^{2} \\
& =\left|\int_{0}^{1} \omega^{\frac{1}{2 m-1}-2 d} \nabla \hat{f}\left(\frac{\xi-N_{1}+t\left(N_{2}-N_{1}\right)}{\omega}\right) \cdot\left(N_{2}-N_{1}\right) d t\right|^{2} \\
& \leq \omega^{\frac{2}{2 m-1}-4 d}\left|N_{2}-N_{1}\right|^{2}\left(\int_{0}^{1}\left\|\nabla \hat{f}\left(\frac{\xi-N_{2}+t\left(N_{2}-N_{1}\right)}{\omega}\right)\right\| d t\right)^{2} .
\end{aligned}
$$

Let

$$
\omega=|N|^{\frac{-2 k(2 m-1)}{2-d(2 m-1)}}, \quad N_{1}, N_{2} \simeq N .
$$

We have that $\widehat{f_{\omega}}$ concentrates in $B_{\omega}(0)$. Here we consider two cases:

1. $\mathrm{k}<\frac{\mathrm{d}}{2}-\frac{1}{2 \mathrm{~m}-1}$.

In this case, from (3.8), the exponent of $|N|$ in (4.13) is less than 1 ; then if $\xi \in B_{\omega}( \pm N),|\xi| \simeq N$. It follows, using (4.12), that

$$
\begin{aligned}
& \left\|u_{N_{1}, \omega}(0)-u_{N_{2}, \omega}(0)\right\|_{k}^{2}=\int\left(1+|\xi|^{2}\right)^{k}\left|\widehat{f_{\omega}}\left(\xi-N_{1}\right)-\widehat{f_{\omega}}\left(\xi-N_{2}\right)\right|^{2} d \xi \\
& \leq c|N|^{2 k} \omega^{\frac{2}{2 m-1}-4 d}\left|N_{2}-N_{1}\right|^{2} \int_{\mathbb{R}^{d}}\left(\int_{0}^{1}\left\|\nabla \hat{f}\left(\frac{\xi-N_{2}+t\left(N_{2}-N_{1}\right)}{\omega}\right)\right\| d t\right)^{2} d \xi \\
& \leq c|N|^{2 k} \omega^{\frac{2}{2 m-1}-4 d}\left|N_{2}-N_{1}\right|^{2} \int_{\mathbb{R}^{d}} \int_{0}^{1}\left\|\nabla \hat{f}\left(\frac{\xi-N_{2}+t\left(N_{2}-N_{1}\right)}{\omega}\right)\right\|^{2} d t d \xi \\
& =c|N|^{2 k} \omega^{\frac{2}{2 m-1}-3 d}\left|N_{2}-N_{1}\right|^{2} \int_{0}^{1} \int_{\mathbb{R}^{d}}\left\|\nabla \hat{f}\left(\eta+\frac{-N_{2}+t\left(N_{2}-N_{1}\right)}{\omega}\right)\right\|^{2} d \eta d t \\
& =|N|^{2 k} \omega^{\frac{2}{2 m-1}}-3 d\left|N_{2}-N_{1}\right|^{2}\|\nabla \hat{f}\|^{2} \\
& =|N|^{\frac{4 k d(2 m-1)}{2-d(2 m-1)}}\left|N_{2}-N_{1}\right|^{2}\|\nabla \hat{f}\|^{2} .
\end{aligned}
$$

We have

$$
\begin{aligned}
\left\|u_{N_{j}, \omega}(0)\right\|_{k}^{2} & =\int\left(1+|\xi|^{2}\right)^{k}\left|\widehat{f_{\omega}}\left(\xi-N_{j}\right)\right|^{2} d \xi \\
& \simeq|N|^{2 k} \omega^{\frac{2}{2 m-1}}-2 d \int\left|\hat{f}\left(\frac{\xi-N_{j}}{\omega}\right)\right|^{2} d \xi \\
& =|N|^{2 k} \omega^{\frac{2}{2 m-1}}-d \int\left|\hat{f}\left(\eta-\frac{N_{j}}{\omega}\right)\right|^{2} d \eta \\
& =\|f\|^{2} .
\end{aligned}
$$

Given $T>0$ and $\delta>0, N_{1}, N_{2}$ are chosen so that

$$
T\left|N_{1}-N_{2}\right|>>\omega^{-1}=|N|^{\frac{2 k(2 m-1)}{2-d(2 m-1)}}
$$

and thus there is no interaction of $u_{N_{j}, \omega}, j=1,2$, at time $t=T$ where they are concentrated. Taking

$$
\left|N_{2}-N_{1}\right|=\delta|N|^{\frac{-2 k d(2 m-1)}{2-d(2 m-1)}}
$$


we get from (4.14)

$$
\left\|u_{N_{1}, \omega}(0)-u_{N_{2}, \omega}(0)\right\|_{k}^{2} \leq c \delta^{2}
$$

while

$$
\left\|u_{N_{1}, \omega}(T)-u_{N_{2}, \omega}(T)\right\|_{k}^{2} \simeq\left\|u_{N_{1}, \omega}(T)\right\|_{k}^{2}+\left\|u_{N_{2}, \omega}(T)\right\|_{k}^{2} \geq c|N|^{2 k} \omega^{\frac{2}{2 m-1}-d}=c .
$$

Now we take $|N|$ so large that

$$
|N|^{\frac{-2 k(2 m-1)(d+1)}{2-d(2 m-1)}}>>\frac{1}{T \delta}
$$

which is possible since

$$
k>0, d>\frac{2}{2 m-1},
$$

according our hypotheses in Proposition 3.3 .

The initial data $n_{\omega}(0)$ satisfies, from (4.11),

$$
\begin{aligned}
\left\|n_{\omega}(0)\right\|_{\ell}^{2} & =\int\left(1+|\xi|^{2}\right)^{\ell}\left|\mathcal{F}\left(\left(f_{\omega}\right)^{2 m}\right)(\xi)\right|^{2} d \xi \\
& =\omega^{\frac{4 m}{2 m-1}-2 d} \int\left(1+|\xi|^{2}\right)^{\ell}\left|\widehat{f^{2 m}}\left(\frac{\xi}{\omega}\right)\right|^{2} d \xi \\
& =\omega^{\frac{4 m}{2 m-1}-d} \int\left(1+\omega^{2}|\eta|^{2}\right)^{\ell}\left|\widehat{f^{2 m}}(\eta)\right|^{2} d \eta \\
& \simeq \omega^{\frac{4 m}{2 m-1}+2 \ell-d}\left\|f^{2 m}\right\|_{\ell}^{2} \\
& \simeq|N|^{\frac{-2 k(4 m+(2 m-1)(2 \ell-d))}{2-d(2 m-1)}}\left\|f^{2 m}\right\|_{\ell}^{2} \\
& \leq\left\|f^{2 m}\right\|_{\ell}^{2}
\end{aligned}
$$

since the exponent of $|N|$ above is $\leq 0$ in both cases of Propositions 2.2 and 3.3

2. $\mathrm{k}=\frac{\mathrm{d}}{\mathbf{2}}-\frac{\mathbf{1}}{\mathbf{2 m - 1}}$.

Take $\omega=|N|^{\alpha}$, for some $\alpha>1$. Then if $\xi \in B_{\omega}( \pm N)$, then $|\xi| \simeq|N|^{\alpha}$ and

$$
\left\|u_{N, \omega}(0)\right\|_{k}^{2} \simeq \omega^{2 k} \omega^{\frac{2}{2 m-1}-d}=c|N|^{2 \alpha\left(k+\frac{1}{2 m-1}-\frac{d}{2}\right)}=c .
$$

Analogously

$$
\begin{aligned}
\left\|u_{N_{1}, \omega}(0)-u_{N_{2}, \omega}(0)\right\|_{k}^{2} & \leq c|N|^{2 k \alpha} \omega^{\frac{2}{2 m-1}-3 d}\left|N_{2}-N_{1}\right|^{2} \\
& =c|N|^{\alpha\left(2 k+\frac{2}{2 m-1}-3 d\right)}\left|N_{2}-N_{1}\right|^{2} .
\end{aligned}
$$

Again, if we take

$$
T\left(N_{2}-N_{1}\right)>>\omega^{-1}=|N|^{-\alpha},
$$

there is no interaction of $u_{N_{j}, \omega}, j=1,2$, at time $t=T$ where they are concentrated. Thus

$$
\left\|u_{N_{1}, \omega}(T)-u_{N_{2}, \omega}(T)\right\|_{k}^{2}=\left\|u_{N_{1}, \omega}(T)\right\|_{k}^{2}+\left\|u_{N_{2}, \omega}(T)\right\|_{k}^{2} \geq c .
$$

If we take

$$
\left|N_{2}-N_{1}\right|=\delta|N|^{-\frac{\alpha}{2}\left(2 k+\frac{2}{2 m-1}-3 d\right)},
$$

then

$$
\left\|u_{N_{1}, \omega}(0)-u_{N_{2}, \omega}(0)\right\|_{k}^{2} \leq c \delta^{2}
$$


Choosing $|N|$ large enough so that

$$
|N|^{-\frac{\alpha}{2}\left(2 k+\frac{2}{2 m-1}-3 d\right)+\alpha}>>\frac{1}{T \delta},
$$

which is possible since

$$
\begin{aligned}
-\frac{\alpha}{2}\left(2 k+\frac{2}{2 m-1}-3 d\right)+\alpha & =-\frac{\alpha}{2}\left(d-\frac{2}{2 m-1}+\frac{2}{2 m-1}-3 d\right)+\alpha \\
& =\alpha(d+1)>0,
\end{aligned}
$$

we get (4.16). For the initial data $n_{\omega}(0)$ we have

$$
\begin{aligned}
\left\|n_{\omega}(0)\right\|_{\ell}^{2} & \simeq \omega^{\frac{4 m}{2 m-1}+2 \ell-d}\left\|f^{2 m}\right\|_{\ell}^{2} \\
& \simeq|N|^{\alpha\left(\frac{4 m}{2 m-1}+2 \ell-d\right)}\left\|f^{2 m}\right\|_{\ell}^{2} \\
& \leq\left\|f^{2 m}\right\|_{\ell}^{2}
\end{aligned}
$$

since $\ell \leq \frac{d}{2}-\frac{2 m}{2 m-1}$. This finishes the proof of Proposition 3.3

Proof of Proposition 2.2. For the one-dimensional Zakharov system (2.2) $d=m=$ 1 , notice that the same argument of the proof of Proposition 3.3, case 1, follows using the facts:

a) the exponent of $|N|$ in (4.13) is less than 1 provided $k>-1 / 2$;

b) the exponent of $|N|$ in (4.15D) is $>0$ if $k<0$.

For the case $k \leq-1 / 2$ we use [6].

\section{REFERENCES}

1. H. Added and S. Added, Existence globale de solutions fortes pour les équations de la turbulence de Langmuir em dimension 2, C. R. Acad. Sci. Paris 299 (1984), 551-554. MR 86g:35163

2. H. Added and S. Added, Equations of Langmuir turbulence and nonlinear Schrödinger equation: smoothness and approximation, J. Funct. Anal. 79 (1988), 183-210. MR 89h:35273

3. H. Berestycki and P. L. Lyons, Nonlinear scalar field equations, Arch. Rational Mech. Anal. 82 (1983), 313-376. MR 84h:35054a MR 84h:35054b

4. H. A. Biagioni and F. Linares, Ill-posedness for the derivative Schrödinger and generalized Benjamin-Ono equation, Trans. Amer. Math. Soc. 353 (2001), 3649-3659. MR 2002e:35215

5. B. Birnir, C. E. Kenig, G. Ponce, N. Svanstedt and L. Vega, On the ill-posedness of the IVP for the generalized Korteweg-de Vries and nonlinear Schrödinger equations, J. London Math. Soc. (2) 53 (1996), 551-559. MR 97d:35233

6. B. Birnir, G. Ponce and N. Svanstedt, The local ill-posedness of the modified KdV equation. Ann. Inst. H. Poincaré Anal. Non Linéaire 13 (1996), no. 4, 529-535. MR 97e:35152

7. J. Bourgain and J. Colliander, On well-posedness of the Zakharov system, Int. Math. Res. Not. 11 (1996), 515-546. MR 97h:35206

8. J. Colliander, Wellposedness for Zakharov systems with generalized nonlinearity, J. Differential Equations 148 (2), (1998), 351-363. MR 99h:35196

9. J. Ginibre, Y. Tsutsumi and G. Velo, On the Cauchy problem for the Zakharov system, J. Funct. Anal 151 (1997), 384-436. MR 2000c:35220

10. C.E. Kenig, G. Ponce and L. Vega, On the Zakharov and Zakharov-Schulman systems, J. Funct. Anal. 127 (1995), 204-234. MR 96a:35197

11. C.E. Kenig, G. Ponce and L. Vega, On ill-posedness of some canonical dispersive equations, Duke Math. J., 106 (2001), 617-633. MR 2002c:35265

12. T. Ozawa and Y. Tsutsumi, Existence and smoothing effect of solutions for the Zakharov equations, RIMS Kyoto Univ. 28 (1992), 329-361. MR 93k:35246

13. W. Strauss, Existence of solitary waves in higher dimensions, Comm. Math. Phys. 55 (1977), 149-162. MR 56:12616

14. C. Sulem and P. L. Sulem, Quelques résultats de régularité pour les équations de la turbulence de Langmuir, C. R. Acad. Sci. Paris 289 (1979), 173-176. MR 80i:35165 
15. Y. Wu, Orbital stability of solitary waves of Zakharov system, J. Math. Phys. 35 (1994), 2413-2422. MR 95e:35170

16. V.E. Zakharov, The collapse of Langmuir waves, Sov. Phys. JETP 35 (1972), 908-914.

Departamento de Matemática, imeCC-UniCAMP, 13081-970, Campinas, SP, Brasil

E-mail address: hebe@ime.unicamp.br

Instituto de Matemática Pura e Aplicada, 22460-320, Rio de Janeiro, Brasil

E-mail address: linares@impa.br 